\title{
Molecular Characterization of Factor V Leiden G1691A and Prothrombin G20210A Mutations in Saudi Females with Recurrent Pregnancy Loss
} Gihan E-H Gawish ${ }^{1,2}$ and Osama Al-Khamees ${ }^{2}$

${ }^{* 1}$ Oral Biological and Medical Science, University of British Columbia, P.O. Box V6T 1Z4, Vancouver, Canada

${ }^{2}$ Collage of Medicine, Imam Muhammad Bin Saud University, Riyadh, Saudi Arabia P.O. Box 7897-232, Riyadh 11159, Kingdom of Saudi Arabia

\begin{abstract}
Recurrent pregnancy loss (RPL) is the most common complication of pregnancy in Saudi Female. Approximately $18 \%$ of all clinically recognized pregnancies are spontaneously aborted. Although several causes of RPL have been established, more than $50 \%$ of cases remain unexplained. Recently, thrombophilias have been suggested as a possible cause of RPL. Factor V Leiden (FVL) (G1619A) and Factor II (prothrombin) (G20210A) gene mutations are the most common types of hereditary thrombophilias, but are usually undiagnosed because most carriers are asymptomatic. The aim of this study was to investigate two common mutations of the factor V Leiden (G1619A) and factor II Prothrombin (G20210A) gene mutations and to assess whether recurrent pregnancy loss patterns among Saudi women differ according to causal/associated conditions. The study including 142 females, 72 had a history of 2 or more events of fetal loss in any of the 3 trimesters of pregnancy. The other 70 were clinically healthy women with a good obstetric history taken as a control group. Detection of FV Leiden (G1691A) and FII (Prothrombin G20210A) mutations were done using multiplex allele-specific PCR amplification. The results indicated that the total mutation carriage rate (AA and AG) among cases for FII was higher in frequency than FVL. Both were significantly higher than controls $P>0.0001$ while FIIP>0.0001. The frequencies of FVL \& FII mutations related to the pregnancy loss stages showed that FVL mutation ratio was high among cases with early pregnancy loss $(26 \%)$ followed by the late stage $(25 \%)$ and controls $(1.4 \%)$ that was statistically significant. On the other hand FII mutation ratio was high among cases with late pregnancy loss $(50 \%)$ followed by early $(38 \%)$ and controls $(1.4 \%)$ that was statistically significant. We concluded that there are a strong association between the presence of thrombophilic mutations related to FVL and FII genes among Saudi women. Identifying heritable thrombophilia women might potentially prevent miscarriages, as well as serious maternal and neonatal complications.
\end{abstract}

Keywords: Recurrent pregnancy loss; Late pregnancy loss; Thrombophilia; Factor V Leiden; Prothrombin

Abbreviation: FV: Factor V; WT: Wild Type; PT: Prothrombin; FIX: Factor IX.

\section{Introduction}

Recurrent pregnancy loss (RPL), defined as 2 or more spontaneous abortions, affects approximately 5\% of women of reproductive age. Although several causes of RPL have been established, more than 50\% of cases remain unexplained. This is a challenging dilemma for both patients and physicians. Recently, thrombophilias have been suggested as a possible cause of RPL [1]. The aim of this study was to investigate two common mutations of the factor V Leiden (G1619A) and factor II Prothrombin (G20210A) gene mutations and to assess whether recurrent pregnancy loss patterns among Saudi women differ according to causal/associated conditions.

Thrombophilia is considered still a debated problem that may be common in women with unexplained recurrent pregnancy loss, with prevalence as high as $65 \%$ in selected populations $[2,3]$. The thrombophilias are a number of prothrombotic factors, which can either be inherited or acquired. The inherited thrombophilias include activated protein $\mathrm{C}$ resistance $[95 \%$ due to factor $\mathrm{V}$ Leiden (FVL) mutation], protein $\mathrm{S}$ deficiency, protein $\mathrm{C}$ deficiency, antithrombin III deficiency, FII (prothrombin) mutation and hyperhomocysteinaemia $[1,4,5]$ Factor V Leiden (FVL) and prothrombin (G20210A) mutations are the 2 most common causes have been implicated as risk factors of hereditary thrombophilias which in turn can result in placentation.

Early in pregnancy, this may manifest as spontaneous loss. In later pregnancy thrombophilias have been associated with complications such as pre-eclampsia, intrauterine growth restriction, placental abruption, and stillbirth. Most carriers of the mutation do not develop any clinical signs and remain undiagnosed because conditions result in a small absolute risk of clinically significant thrombosis. However, when one exposed to additional risk factors those promote stasis, such as pregnancy or possible oral contraceptives, or hormone replacement therapy, and disorders of the vessel wall. The risk threatening thrombotic events is significantly increased and may become clinically evident $[6,7]$ The FVL mutation is the most studied inherited thrombophilia in relation to pregnancy complications. Factor V Leiden is a genetic disorder was described as an underlying cause of APC (Anti Protein $\mathrm{C})$ resistance. This mutation involves a guanine to adenine substitution (factor V Arg506Gln or factor V Leiden) (G-to-A) at nucleotide 1691 in exon 10, which results in synthesis of a defective factor $\mathrm{V}$ molecule (FVL), resistant to cleavage by APC [8-10]. On the other hand, prothrombin is the precursor molecule of thrombin. Sequence variation of a $G$ to $A$ transposition in position 20210 of the prothrombin gene recently was

*Corresponding author: Gihan E-H Gawish, Oral Biological and Medical Science, University of British Columbia, P.O. Box V6T 1Z4, Vancouver, Canada; E-mail: ggawish@ksu.edu.sa

Received August 03, 2013; Accepted October 10, 2013; Published October 15, 2013

Citation: Gawish G, Al-Khamees O (2013) Molecular Characterization of Factor V Leiden G1691A and Prothrombin G20210A Mutations in Saudi Females with Recurrent Pregnancy Loss. J Blood Disorders Transf 4: 165. doi: 10.4172/21559864.1000165

Copyright: (c) 2013 Gawish G, et al. This is an open-access article distributed under the terms of the Creative Commons Attribution License, which permits unrestricted use, distribution, and reproduction in any medium, provided the original author and source are credited. 
identified as a genetic risk factor for thrombosis. This mutation results in increased plasma prothrombin and a twofold to four fold increased risk for thromboembolic disease $[2,11]$.

Gihan E-H Gawish [12] found that Factor II (prothrombin) and Factor V Leiden mutations, however, may be important risk factors for neonatal stroke in Saudi newborns, She recommended that Factor V Leiden G1691A and prothrombin G20210A mutations are important and must be included in the routine analysis of Saudi fetuses and pregnant women.

\section{Materials and Methods}

Genomic DNA samples of 142 Saudi women, who recruited and followed at Military Hospitals in Saudi Arabia under human ethical approval, were screened from Dec. 2009 to Dec. 2010. 70 out of them were healthy females as control group with negative personal and negative family history for pregnancy loss and also with negative history to any thromboebolic diseases. They have at least one previous uneventful pregnancy. While other 72 females having history of recurrent pregnancy loss 2 times or more of abortions, miscarriages, intrauterine fetal death (IUFD) or still birth associated with placental vessel thrombosis or placental infarction on histopathology examination. Exclusion criteria included cases diagnosed with genetic/ chromosomal aberrations, anatomic anomalies, and or endocrine/ hormonal abnormalities.

Laboratory investigations included liver function tests, blood sugar. immunological markers (antiphospholipid, anticardiolipin), TORCH (toxoplasma gondii, rubella, cytomegalovirus, Herpes Simplex), complete blood count and coagulation profile (bleeding, clotting time, the routine evaluation for prothrombotic conditions included plasminogen, antithrombin, protein $\mathrm{C}$, free protein $\mathrm{S}$, activated protein $\mathrm{C}$ resistance) were be done. Factor V Leiden (G1691A) and the prothrombin gene mutations (G20210A mutation) were detected using a multiplex allele-specific PCR amplification.

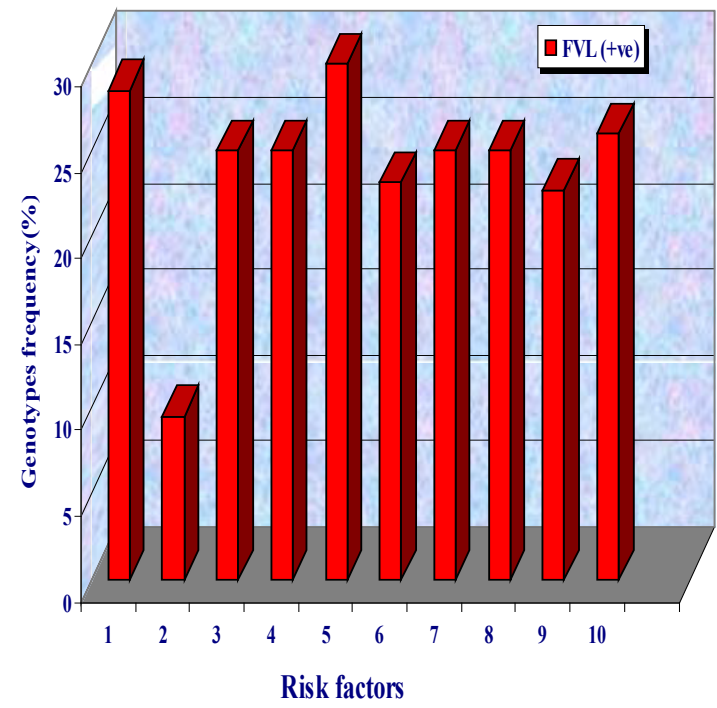

Figure 1: Frequency of demographic risk factors in relation to factor $V$ (Leiden) mutation among cases of recurrent pregnancy loss; FVL (+ve)=GA\&AA. The figure shows Age $<30$, Age $>30$, Rh (+ve), Rh (-ve), Blood Gp (+O), Blood Gp $(-\mathrm{O})$, Passive smoking (+ve), Passive smoking (+ve), Infection (+ve), Infection (-ve) respectively

\section{Primer design}

Primers were designed by using a web-based PRIMER 3.0 program (http://workbench.sdsc.edu). We used the "BLAST" program at http:// www.ncbi.nlm.nih.gov/blast to check for the specificity of the primers.

\section{PCR method}

Genomic DNA was isolated from the peripheral blood samples according to a standard protocol (Qiagen- Germany). DNA pellet was dissolved in $50 \mu \mathrm{l}$ of TE buffer $(\sim 30 \mathrm{ng} / \mu \mathrm{l}$ DNA concentration $)$ and stored at $-20^{\circ} \mathrm{C}$ until the genotype analysis was performed.

Detection of Factor V Leiden (G1691A) and Prothrombin (G20210A) Gene Mutation:

Factor V Leiden (G1691A) and Prothrombin (G20210A) gene mutation were analyzed using a multiplex allele-specific PCR amplification method of Ranguelov et al. [13] all samples were subjected to amplification of a region of the factor IX gene as an internal control for assessment of the quality of the extracted DNA. Each PCR was performed with 300ng of DNA, $200 \mu \mathrm{mol} / \mathrm{L}$ of each $\mathrm{dNTP}, 500 \mathrm{nmol} / \mathrm{L}$ of each primer, and 2.5 units of Taq DNA polymerase (Amplitaq Gold, Perkin-Elmer Cetus, Norwalk, Conn). DNA was initially denatured for 10 minutes at $95^{\circ} \mathrm{C}$, and then 10 cycles were performed as follows: $94^{\circ} \mathrm{C}$ for 30 seconds, $60^{\circ} \mathrm{C}$ for 30 seconds, and $72^{\circ} \mathrm{C}$ for 1 minute. Then, 25 cycles were performed as follows: $94^{\circ} \mathrm{C}$ for 30 seconds, $55^{\circ} \mathrm{C}$ for 30 seconds, and $72^{\circ} \mathrm{C}$ for 1 minute. The PCR amplification was completed by a final extension at $72^{\circ} \mathrm{C}$ for 7 minutes. Amplification of the factor $\mathrm{V}$ and prothrombin genes resulted in 152 base pair (bp) and 340-bp products, respectively. The internal amplification control (ie, the region of the factor IX gene) resulted in a 250-bp product. The amplified products were then electrophoresed in $2 \%$ agarose gel, stained with ethidium bromide, and visualized under UV light. The sequences of primers are as follow: FV common (5'-GGA CTA CTT GAC AAT TAC TGT TCT CTT G-3'), FV WT(5'-GCA GAT CCC TGG ACA GAC G-3'), FV mutant (5'-GCA GAT CCC TGG ACA GAC A-3', PT common (5'-TCT AGA AAC AGT TGC CTG GCA G-3'), PT WT (5'GCA CTG GGA GCA TTG AGG ATC-3'), PT mutant (5'-GCA CTG GGA GCA TTG AGG ATT-3'), FIX-1 (5'-CTC CTG CAG CAT TGA GGG AGA TGG ACA TT-3') and FIX-2 (5'-CTC GAA TTC GGC AAG CAT ACT CAA TGT AT-3').

Data were analyzed using the SPSS program. Power calculations were performed to obtain the probability that differences in gene frequencies were statistically significant. $P$ values $>0.05$ were considered significant, $>0.01$ highly significant, and $>0.0001$ extremely significant. Odds ratios were also calculated for the samples

\section{Result}

The participants included 142 women subjects. Out of them, 72 had a history of 2 or more events of recurrent fetal loss (abortion, miscarriage or still birth). Their mean age \pm SD was $25.13 \pm 4.58$. The mean age of healthy women was $34.4 \pm 4.88$. $72.22 \%$ cases were early aborted, $8.33 \%$ late aborted, $22.22 \%$ early and late aborted. Four $5.55 \%$ were diabetic, $27.77 \%$ had a history of passive smoking, $4.16 \%$ were hypertensive and $30.55 \%$ had a positive. TORCH infection (Toxoplasma Gondii, Rubella, Cytomegalovirus, Herpes Simplex) manifested by high levels of corresponding antibodies of IgM type.

The results have showed that Factor V (Leiden) carriage genotypes (AA\& GA) mutations frequency was higher among cases with low age group $<30$ years than those with high age group $>30$ year $(28.5 \%$ vs. $9.5 \%$ respectively). However there wasn't any significant association 
Citation: Gawish G, Al-Khamees O (2013) Molecular Characterization of Factor V Leiden G1691A and Prothrombin G20210A Mutations in Saudi Females with Recurrent Pregnancy Loss. J Blood Disorders Transf 4: 165. doi: 10.4172/2155-9864.1000165

Page 3 of 7

between age of the cases as risk factor and presence of factor $\mathrm{V}$ (Leiden) mutation $(\mathrm{P}=0.07)$. Similarly, there was insignificant association between cases and other risk factors subgroups related to $\mathrm{Rh}, \mathrm{ABO}$ blood grouping, history of passive smoking and infection(TORCH) $(\mathrm{P}>0.05)$, regarding in its effect among studied cases and showed no association with presence or absence of factor $\mathrm{V}$ (Leiden)mutation (Figure 1). Also, Factor II (Prothrombin) carriage genotypes (AA\& GA) mutations frequency was significantly higher among studied cases with passive smoking compared with negative cases[62.5\% vs. $25 \%$, $\mathrm{P}=0.003]$. To the contrary of that, the other studied subgroups related to $[\mathrm{Rh}, \mathrm{ABO}$ blood grouping, age and infection (TORCH)] showed no significant association with factor II (Prothrombin) mutation (Figure 2). Also, There is a statistically significant higher frequency among cases with history of passive smoking and carriage of factor II either heterozygous (GA) or homozygous (AA) genotypes mutation than

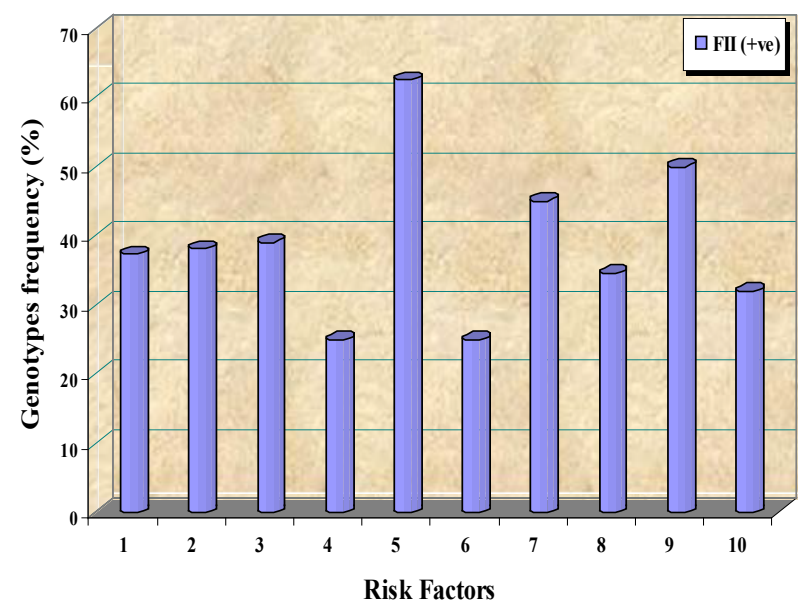

Figure 2: Frequency of demographic risk factors in relation to factor I mutation among cases of recurrent pregnancy loss; FII (+ve)=GA\&AA. The figure shows Age $<30, A g e>30, R h(+v e)$, Rh (-ve), Blood Gp (+O), Blood Gp $(-\mathrm{O})$, Passive smoking (+ve), Passive smoking (+ve), Infection (+ve), Infection (-ve) respectively.

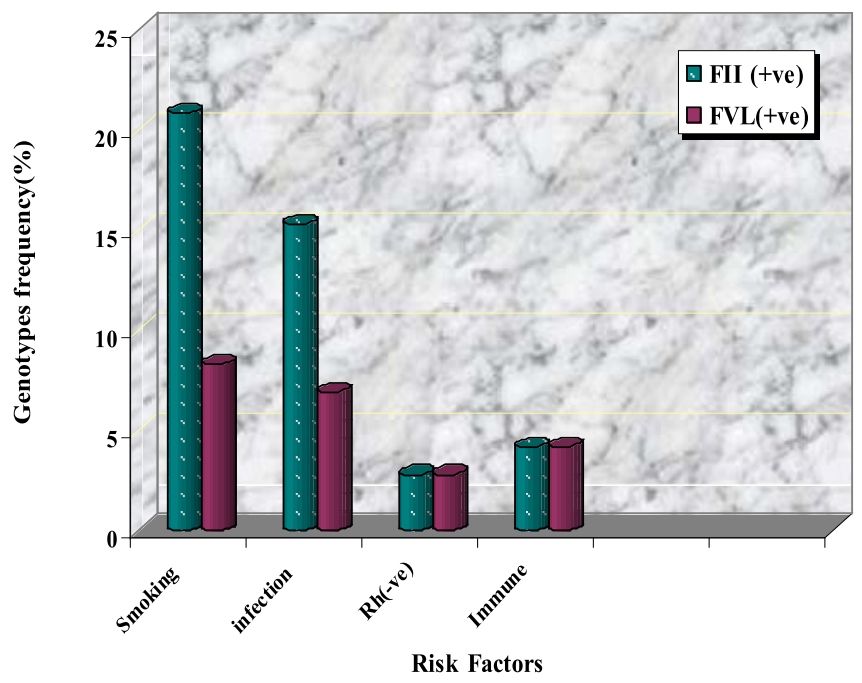

Figure 3: Frequency of demographic risk factors in relation to factor $\mathrm{V}$ (Leiden) compared to factorll mutation among cases of recurrent pregnancy loss; FVL $(+v e)=$ GA\&AA and FII (+ve)= GA\&AA.

\begin{tabular}{|c|c|c|c|c|}
\hline Classes of recurrent abortion (n) & $\begin{array}{l}\text { FV GG/FII } \\
\text { GA n (\%) }\end{array}$ & $\begin{array}{l}\text { FV GA/FII } \\
\text { GG n (\%) }\end{array}$ & $\mathbf{X}^{2}$ & P \\
\hline Early (50) & $14(28)$ & $8(16)$ & & \\
Late (8) & $3(37.5)$ & $1(12.5)$ & 0.9 & 0.24 \\
Early+Late (14) & $3(21.4)$ & $2(14.3)$ & & \\
\hline
\end{tabular}

$\mathrm{n}=$ number of cases, GG homozygous wild type, GA heterozygous, AA homozygous mutant, Early $=0-20$ weeks Late $=20-40$ weeks

Table 1: Combined genotypes of Factor V (Leiden) \& Factor II (Prothrombin) of studied cases related to classes of recurrent abortion.

\begin{tabular}{|l|c|c|c|c|}
\hline Genotype & $\begin{array}{c}\text { Cases } \\
\mathbf{7 2}(\mathbf{1 0 0})\end{array}$ & $\begin{array}{c}\text { Controls } \\
\mathbf{7 0}(\mathbf{1 0 0})\end{array}$ & $\mathbf{P}$ & OR (95\%CI) \\
\hline Individual genotype frequency & & \\
\hline GG & $50(69.44)$ & $68(97.14)$ & $<0.0001^{* *}$ & $0.03(0.0-0.44)$ \\
\hline GA & $15(20.83)$ & $2(2.86)$ & $<0.0001^{* *}$ & $19.38(1.75-155.37)$ \\
\hline AA & $2(2.78)$ & $0(0)$ & 1 & $2.96(0.12-73.91)$ \\
\hline Total homozygous \&heterozygous mutant \\
\hline AA+GA & $17(23.6)$ & $1(1.43)$ & $<0.0001^{* *}$ & $23(2.97-177.85)$ \\
\hline Individual allele frequency & & & \\
\hline A & $19(13.19)$ & $1(0.71)$ & $<0.0001^{* *}$ & $21.13(2.79-160.2)$ \\
\hline G & $125(86.81)$ & $139(99.29)$ & $<0.0001^{* *}$ & $0.05(0.0-0.36)$ \\
\hline
\end{tabular}

GG: homozygous wild type, GA: heterozygous, AA: homozygous mutant $P$ : probability, OR: Odds Ratio, $\mathrm{Cl}$ : Confidence Intervals,

${ }^{\star *} \mathrm{P}<0.0001$ extremely significant

Table 2: Frequency of factor $V$ (Leiden) mutation among cases of recurrent pregnancy loss compared to controls.

those carriage factor $\mathrm{V}$ either homozygous (AA) or heterozygous (GA) genotypes ( $20.8 \%$ vs. $8.3 \%, \mathrm{P}=0.03$ respectively). Similarly cases carriage factor II positive and suffer from (TORCH) infection were high frequency than those carriage factor $\mathrm{V}$ (Leiden) and TORCH, but insignificant $(\mathrm{P}=0.11)$. On the other hand, there was no significant association between cases carriage any of both mutation and other risk factors subgroups related to $\mathrm{Rh}$, immune factor $(\mathrm{P}=1)$ (Figure 3 ).

\section{Genotyping}

Early abortion cases having FII (GA) heterozygous mutation were higher than those having FVL (GA) heterozygous mutation (28\% vs. $16 \%$ ). The same was observed among cases either with (late or combined late and early abortion), but statistically insignificant $(\mathrm{P}=0.9)$ (Table 1).

A multiplex PCR was established to detect Factor V Leiden (G1691A) and prothrombin (G20210A) mutations. The effects of several factors (including $\mathrm{MgCl} 2$ concentration, primer concentration, and PCR cycling conditions) on PCR specificity and efficiency were determined and optimized. For Factor V Leiden, there is statistically significant lower frequency of homozygous normal genotype (GG) among cases compared to controls [69.44\% vs. 97.14\%, P<0.0001]. On the other hand, they disclosed a significant higher frequency of the heterozygous mutant form (GA) than controls [20.83\% vs. $2.86 \%$, $\mathrm{P}<0.0001]$. Thus, the total carriage rate of FVL, A mutation represented in both heterozygote form (GA) and homozygote (AA) mutant genotype frequencies was significantly higher among cases versus controls [23.6\% vs. $1.43 \%, \mathrm{P}>0.0001]$. Regarding allelic frequencies, the mutant A allele was significantly higher in cases than in controls [ $13.19 \%$ vs. $0.71 \%, \mathrm{P}>0.0001$ ]; the reverse was noted with the wild type allele $\mathrm{G}$ which was significantly lower among cases compared to controls (85.81\% vs. 99.29\%) (Table 2 and Figure 4).

With regard to prothrombin, there is a statistically significant lower frequency of homozygous normal genotype (GG) among cases compared to controls [61.1\% vs. $95.5 \%, \mathrm{P}<0.0001]$. On the other hand they showed a significant higher frequency of the heterozygous mutant form (GA) than controls $[34.72 \%$ vs. $1.43 \%, \mathrm{OR}=36.7,95 \% \mathrm{CI}=4.8$ 
Citation: Gawish G, Al-Khamees O (2013) Molecular Characterization of Factor V Leiden G1691A and Prothrombin G20210A Mutations in Saudi Females with Recurrent Pregnancy Loss. J Blood Disorders Transf 4: 165. doi: 10.4172/2155-9864.1000165

Page 4 of 7

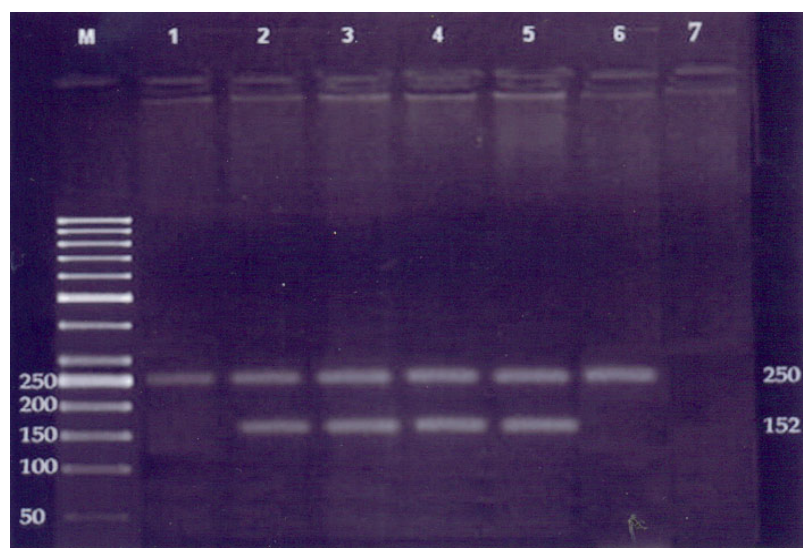

Figure 4: Amplification of FVL (G1691A) using primers for mutant $A$ alleles (lanes $1,3,5$ ) and normal $G$ allele (lanes $2,4,6$ ) showing positive bands (152bp) in lane 2, 3,4,5 with negative bands in lanes 1,6 indicating $G / G$ normal genotype in case 1 (lanes 1,2), G/A heterozygous genotype in case 2 (lanes 3,4), A/A homozygous mutant genotype in case 3 (lanes 5,6) and. M indicates molecular marker, Bands of size 250 bps belongs to F IX used as internal controls.

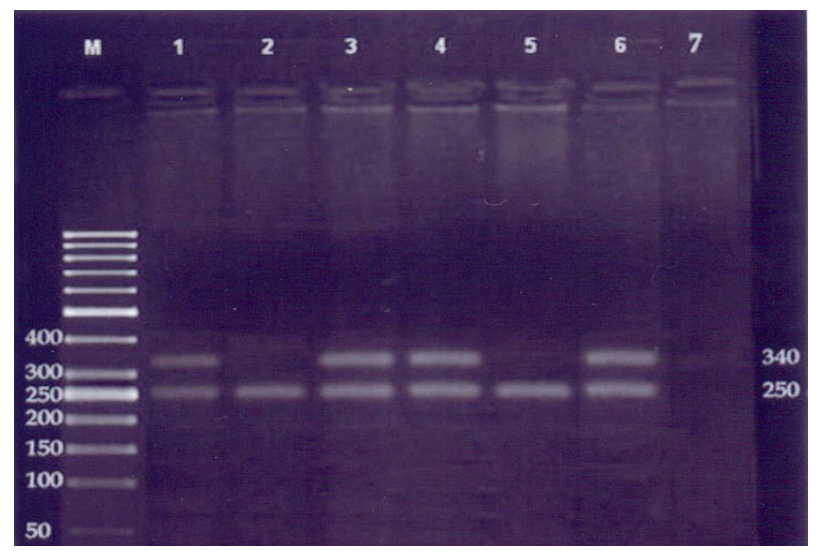

Figure 5: Amplification of FII(G20210A) using primers for mutant $A$ alleles (lanes $1,3,5$ ) and normal $G$ allele (lanes $2,4,6$ ) showing positive bands $(340 \mathrm{bp}$ ) in lane 1, 3, 4, 6 with negative bands in lanes 2,5 indicating $A / A$ homozygous mutant genotype in case 1 (lanes 1,2) and G/A heterozygous genotype in case 2 (lanes 3,4), G/G normal genotype in case 3 (lanes 5,6). M indicates molecular marker, Bands of size 250 bps belongs to F IX used as internal controls.

80.39, $\mathrm{P}<0.0001$ ]; thus, both heterozygote form (GA) and homozygote (AA) mutant genotype frequencies was significantly higher among cases versus controls [30.5\% vs. $1.04 \%, \mathrm{P}>0.0001$ ]. Regarding allelic frequencies, the mutant $\mathrm{A}$ allele was significantly higher in cases than in controls [ $19.14 \%$ vs. $0.71 \%, P>0.0001$ ]; the reverse was noted with the wild type allele $\mathrm{G}$ which was statistically significant lower among cases compared to controls (79.86\% vs. 99.29\%) (Table 3 and Figure 5).

We have reported that there is a statistically significant lower frequency of homozygous normal genotype (GG) of both genes compared to controls $\mathrm{P}<0.0001$. On the contrary cases indicated higher frequency of combined factor II heterozygous mutant form (GA) with homozygous normal factor $\mathrm{V}$ (Leiden) (GG) than controls $\mathrm{P}<0.0001$ and higher frequency of combined heterozygous factor $\mathrm{V}$ (Leiden) with a homozygous normal factor II genotypes (GG) $\mathrm{P}=0.0$ (Figure 6).

\section{Discussion}

In recurrent pregnancy loss, there is much debate about cause and association. However, it is important to note that this is not a uniform finding indeed, many studies have been of a small size, prone to stratification and admixture bias, in which there has been poor matching of cases and controls as a result of racial heterogeneity. In addition, publication bias is evident, as judged by the discrepancy between the number of published abstracts reporting a lack of association between genetic thrombophilia and the number of peerreviewed papers reporting an association $[14,15]$.

Goodman and his colleagues [16] found a significant increased frequency of FVL and FII gene mutation among Chicago American women with history of RPL $(\mathrm{p}<0.0001)$. On the other hand Coulam and colleagues [17] studied Chicago's American women and found no differences in the frequency of specific gene mutations of FVL or FII. However, the prevalence of homozygous mutations and total gene mutations among patients with recurrent miscarriage was significantly higher than among controls. Co-inherited mutations were found in $68 \%$ of women with a history of RPL contrasted to $21 \%$ of control women.

\begin{tabular}{|c|c|c|c|c|}
\hline Genotype & $\begin{array}{c}\text { Cases } \\
\mathrm{N}=72(100 \%)\end{array}$ & $\begin{array}{c}\text { Controls } \\
\mathrm{N}=70(100 \%)\end{array}$ & $\mathbf{P}$ & $\begin{array}{c}\text { OR } \\
(95 \% \mathrm{Cl})\end{array}$ \\
\hline \multicolumn{5}{|c|}{ Individual genotype frequency } \\
\hline GG & $44(61,1)$ & $\begin{array}{c}69 \\
(98.57)\end{array}$ & $<0.0001^{* *}$ & $0.02(0.0-0.18)$ \\
\hline GA & $22(30.5)$ & $1(1.43)$ & $<0.0001^{* *}$ & $36.7(4.8-280.39)$ \\
\hline AA & $2(2.78)$ & $0(0)$ & 0.50 & $5(0.24-106.11)$ \\
\hline \multicolumn{5}{|c|}{ Total homozygous \& heterozygous mutant } \\
\hline$A A+G A$ & $27(37.5)$ & $1(1.43)$ & $<0.0001^{* *}$ & $\begin{array}{c}41.4(5.43- \\
315.68)\end{array}$ \\
\hline \multicolumn{5}{|c|}{ Individual allele frequency } \\
\hline A & \multirow{2}{*}{$28(19.14)$} & $1(0.71)$ & $<0.0001^{* *}$ & $* \quad 35.05(4.7-261.4)$ \\
\hline G & & $139(99.2$ & ) $<0.0001^{* *}$ & ** $0.03(0.0-0.21)$ \\
\hline
\end{tabular}

GG: homozygous wild type, GA: heterozygous, AA: homozygous mutant p: probability, OR: Odds Ratio, Cl: Confidence Intervals,

${ }^{* *} \mathrm{P}<0.0001$ extremely significant

Table 3: Frequency of factor II (Prothrombin) mutation among cases of recurrent pregnancy loss compared to controls.

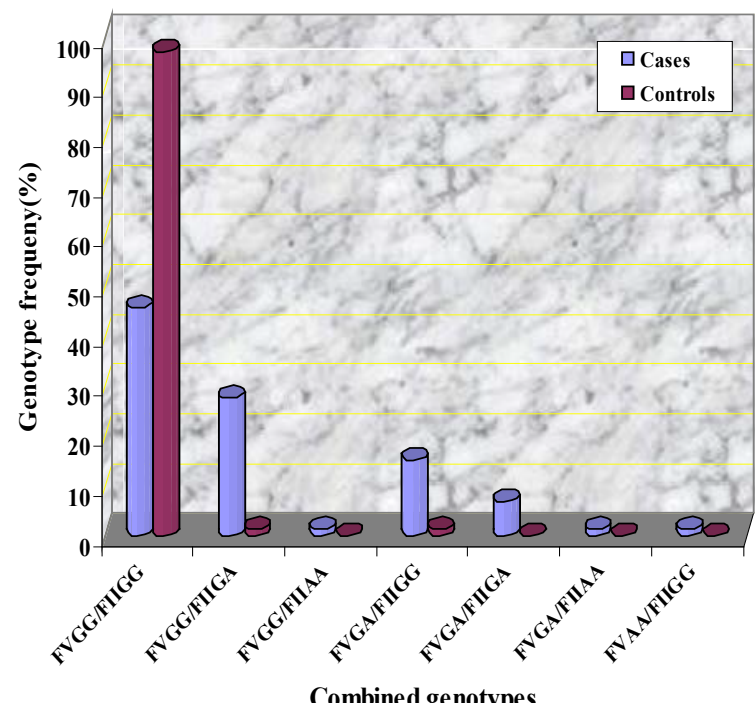

Combined genotypes

Figure 6: Frequency of combined factor $\mathrm{V}$ (Leiden) and factor II mutation among cases of recurrent pregnancy loss compared to controls. 
Finally their conclusion instated that inherited thrombophilias are associated with recurrent miscarriage. This association is manifest by total number of mutations rather than specific genes involved. Another study reported a prevalence of the FVL mutation was greater among case-patients than among controls in American Brigham's women (8.0\% vs. $3.7 \%, \mathrm{OR}=2.3,95 \% \mathrm{CI}=1.0-5.2 ; \mathrm{P}=0.05)[18]$.

Our study revealed a very high risk of pregnancy loss among subjects carrying (GA\& AA) genotypes the mutant allele of FVL and factor II manifested by a very high odds ratio compared to controls. Also, we found that the mutant A allele was significantly higher among cases than in controls both for FVL and for FII gene polymorphisms. We found a higher frequency of combined factor II heterozygous mutant form (GA) with homozygous normal FVL (GG) in cases than, and higher frequency of combined heterozygous FVL with a homozygous normal FII genotypes (GG). Furthermore, the combined heterozygous (GA) genotype of both FVL and FII appeared only among cases $(6.94 \%$ vs. $0 \%, \mathrm{P}=0.05)$.

Our finding were in agreement with those reported in a study among Jewish women who found FVL and factor II were more common in cases compared to controls ( $32 \%$ vs. $10 \%$ and $8 \%$ vs. $4 \%$, $\mathrm{P}<0.0001$ respectively) noting that $7 \%$ out of them were homozygous mutant to factor II compared to none of the controls, $\mathrm{P}=0.012$ [9]. In contradiction to this finding Carp et al. found among Jewish cases that the prevalence of FVL and FII G20210A was lower in the study group than in the control group (3.7\% vs. $6.1 \%$ for FVL and 4.6 vs. $6.1 \%$ for FII respectively); however, the difference was not statistically significant [15]. Also, Another study reported a non-significant increase in FVL mutation was detected among Jewish women $16 \%$ compared with $5 \%$ in the control group, $(\mathrm{P}=0.14)$ [19].

Other studies carried out among Mediterranean countries have revealed also a relatively high mutation rate of FVL like that done by Zammiti et al. [20] among Tunisian women who reported FVL mutation of $19.4 \%$ of patients ( $4.3 \%$ in the homozygous state) and in $5.5 \%$ of controls. Some other studies showed uneventful role of FII unlike FVL, FII was not found to be a risk factor for gestational VTE or RFL. This was showed by Mtiraoui et al. [21] who studied Tunisian patients with $>$ or $=3$ consecutive early, late, or early-late recurrent pregnancy losses, together with age-matched controls. FV Leiden ( $20.54 \%$ vs. $6.06 \%)$, but not FII mutation $(2.74 \%$ vs. $4.04 \%)$ was significantly higher in patients versus controls.

Sehirali et al. [22] observed that FII mutation was significantly higher in Turkish women with RM compared to controls (10.9\% vs. 2.04\%, $\mathrm{P}<0.05)$. Also, Altintas et al. [23] observed that FVL or FII mutation among Turkish women were nonsignificant compared to controls ( $7.9 \%$ vs. $7 \%, \mathrm{P}=0.780)$ and ( 1.7 vs. $1.6 \%, \mathrm{P}=0.931)$ respectively, while the presence both FVL and FII mutations among cases compared to controls $(9.6 \%$ vs. $8.6 \%, \mathrm{P}=0.756)$. In contradiction to this study

No Leiden mutation of FVL was detected in Japanese women with RSA or the controls Kobashi et al. [24]. Otherwise Xu, didn't find FV and FII mutations either in patients or controls. FV and FII gene mutations may be rare in Chinese women, and have no significance in URESA (unexplained recurrent early spontaneous abortion)

The studies among German women confirmed that FVL was significantly more common in cases with RPL compared to controls ( $10 \%$ vs. $2 \%, p=0.02)$, while FII was not associated with RPL [25].

Regarding late or early fetal loss, our study showed that the FVL mutation rate was high among cases with early pregnancy loss (26\%) followed by the late stage (25\%) that was statistically significant from that of controls (1.4\%). On the other hand, FII mutation rate was higher among cases with late pregnancy loss (50\%) followed by early (38\%) which was also statistically significant from that of controls (1.4\%). Furthermore, comparing cases with early abortion regarding the type of associated mutations, we observed a higher frequency of FII (GA) heterozygous mutation than FVL (GA) heterozygous mutation $(28 \%$ vs. $16 \%$ ) which was insignificant. The same was observed among cases with either late or combined late and early abortion. This denotes that the effect of the mutation may not be particular to certain time of pregnancy i.e. have the same effect in association with either early or late pregnancy loss. In a meta-analysis included 31 studies, FVL was associated with early $(\mathrm{OR}=2.01,95 \% \mathrm{CI}=1.13-3.58)$ and late (7.83, 2.83-21.67) recurrent fetal loss. Exclusion of women with other pathologies that could explain fetal loss strengthened the association between FVL and recurrent fetal loss. FII (Prothrombin) mutation was observed with early recurrent $(2.56,1.04-6.29)$ [26].

Pihusch et al. [27] found a higher frequency of factor II mutations which was more prominent than other studied gene polymorphisms in their case series among German populations. This effect was significant in a subgroup with abortions exclusively in the first trimester $(6.7 \%$, vs. $0.8 \%, \mathrm{P}=0.027, \mathrm{OR}=8.5$ ). Also, Krause and his college observed among German women that cases with early fetal loss had FVL and FII mutations of $25 \%$ and $4 \%$ respectively, while cases with late fetal loss had $10 \%$ and $10 \%$ respectively. They concluded that FVL appeared more in early pregnancy loss Krause and his college, Grandone et al. [28,29] among Italian women found a statistically significant difference between women with only early fetal loss vs. those with late events $(\mathrm{P}=0.04)$ was observed. They demonstrated a strong association between FV Leiden and fetal loss. Furthermore, they indicate that late events are more common in these patients.

On the other hand Brenner et al. [9] found that second or third trimester fetal loss were more common cause of pregnancy termination in Jewish women carried either of FVL or FII mutations compared to controls ( $36 \%$ vs. $17 \%$ respectively, $\mathrm{p}=0.0004)$. Also, Martinelli et al. [30] stated that both the FVL and the prothrombin mutations were associated with an approximate tripling of the risk of late fetal loss among young white women in Milan city. In relatively small retrospective study, Kovacheva et al. [31] found that among Bulgarian women that Factor V Leiden was more common in the group of women with fetal loss in second and third trimester (OR-6.25; $\mathrm{P}<0.001)$ and significant protection for RFL in first trimester (OR-0.16; $\mathrm{P}<0.001)$. Mutation of prothrombin was more common in group of women with fetal loss in first trimester compared to the controls ( $28.3 \%$ vs. $11.2 \%$ respectively; $\mathrm{P}=0.009$; OR-3.11). His conclusion was genetic thrombophilic defects are common in women with RFL and are associated with late fetal loss. Foka et al. [32] among Greek women. He suggested that the prevalence of factor V Leiden and prothrombin G20210A mutations is more prominent in second trimester, primary fetal losses and it is independent of the existence of additional pathology predisposing to recurrent fetal losses. Sottilotta et al. [33] found the same prevalence for the FVL and FII (9.1\%, P=NS compared to controls) among Italian women, while women with still birth had FVL and FII mutation $(23.4 \%$ and $19.1 \%$ respectively, $\mathrm{P}<0.0005)$ were highly significant.

Our study revealed that cases carrying FVL mutation (heterozygous GA or homozygous AA) genotypes had slightly higher frequency among cases with no successful pregnancy (primary aborters) compared to those having successful pregnancy (secondary aborters) (26.9\% vs. $20 \%$ respectively) this difference was statistically significant $(\mathrm{p}<0.05)$. 
Citation: Gawish G, Al-Khamees O (2013) Molecular Characterization of Factor V Leiden G1691A and Prothrombin G20210A Mutations in Saudi Females with Recurrent Pregnancy Loss. J Blood Disorders Transf 4: 165. doi: 10.4172/2155-9864.1000165

Page 6 of 7

Therefore genetic thrombophilias cannot be considered an absolute cause of miscarriage or recurrent abortion. This study showed Egyptian women carriage FVL or FII mutations (homo or heterozygous) with RPL $\geq 3$ times had frequency $(22.2 \% \& 27.8 \%$ respectively). In the same time this study revealed that Egyptian women carriage FVL or FII mutations (homo or heterozygous) with RPL $<3$ times had frequency $(25.9 \%$ \& $40.7 \%$ respectively), this difference wasn't statistically significant.

In the subgroup of case-patients with no successful pregnancies, a prevalence of FVL mutation among American Brigham's women was $(9.0 \%$, Odds ratio $=2.6, \mathrm{CI}=1.0-6.7 ; \mathrm{P}=0.048)$. The prevalence of the mutation in those with three or more pregnancy losses $(7.5 \%)$. Ridker et al. [18] also there was no difference in the distribution of factor V Leiden, FII between Greek patients with two and three or more miscarriages Sotiriadis et al. [34] more recently [35] compared frequency of FVL mutation among Caucasian Jewish women. FVL heterozygosity was found among cases of women with 1 sporadic pregnancy loss compared to controls $12.8 \%$ vs. $7.4 \%$ respectively. While heterozygosity of FVL among cases of RPL compared to controls versus (20.5\%vs. $20.4 \%$ respectively). The FVL frequency in cases with 1 sporadic pregnancy loss didn't differ from RPL cases (12.8 \& 20.5\%). In American women reported that thrombophilic disorders were found in up to $20 \%$ of women with normal pregnancies, suggesting additional risk factors required for the development of complications [2].

In conclusion, recurrent pregnancy loss among Saudi pregnant women was strongly associated with thrombophilic mutations related to both FVL and FII. Characteristically, these cases showed a high frequency of factor II mutation. Also we have to note that some of the cases who have got successful pregnancy still had these mutations indicating that other risk factors might play a role in the background of the disorder.

For this reason, we recommend routine screening for thrombophilic mutations for all Egyptian pregnant women in order to setup an appropriate method of prophylaxis against this pregnancy loss disorders. Special emphasis should be directed to cases with three or more losses and deserves serious consideration after two losses, particularly in women over age 35 and in those with a history of subfertility, a previous abortus having a normal karyotype, or a loss occurring after detection of cardiac activity. This study reviews the hereditary causes of RPL and outlines recommended methods for evaluation of patients with RPL.

\section{Conclusion}

We concluded that there are a strong association between the presence of thrombophilic mutations related to FVL and FII genes among Saudi women. This study has developed to assist in the design of a proposed interventional trial of prophylactic anticoagulation in pregnancy for FVL, PT mutations carriers. Identifying heritable thrombophilia women might potentially prevent miscarriages, as well as serious maternal and neonatal complications. For this reason, we recommend the molecular screening for ledien and prothrombin mutations are important and must be included to the routine analysis of Saudi pregnant women to prognosis the might early abortion in Saudi female which help in early therapeutic protocol and reduce the recurrent abortions.

\section{Acknowledgment}

I would like to thank Female Centre for Scientific and Medical Colleges, for their kind help.

\section{Role of the funding source}

Supported by Research Center- Female Centre for Scientific and Medical Colleges, KSU

\section{References}

1. Carrington $B$, Sacks $G$, Regan L (2005) Recurrent miscarriage: pathophysiology and outcome. Curr Opin Obstet Gynecol 17: 591-597.

2. Kujovich JL (2004) Thrombophilia and pregnancy complications. Am J Obstet Gynecol 191: 412-424.

3. Blickstein I (2006) Thrombophilia and women's health: An overview. Obstet Gynecol Clin North Am 33: 347-356.

4. Wouters MG, Nováková IR, Steegers EA (2003) [Thrombophilia and the prevention of thromboembolic complications during pregnancy and the puerperium]. Ned Tijdschr Geneeskd 147: 1060-1066.

5. Doyle NM, Monga M (2004) Thromboembolic disease in pregnancy. Obste Gynecol Clin North Am 31: 319-344, vi.

6. Herman M, Djelmis J, Troselj Z, IvaniseviÄł M (2006) [Pregnancy outcomes in a patient-heterozygous carrier of R506Q mutation of factor $\mathrm{V}$ (Leiden)]. Acta Med Croatica 60: 277-280.

7. Kovalevsky G, Gracia CR, Berlin JA, Sammel MD, Barnhart KT (2004) Evaluation of the association between hereditary thrombophilias and recurrent pregnancy loss: a meta-analysis. Arch Intern Med 164: 558-563.

8. Lane DA, Grant PJ (2000) Role of hemostatic gene polymorphisms in venous and arterial thrombotic disease. Blood 95: 1517-1532.

9. Brenner B, Sarig G, Weiner Z, Younis J, Blumenfeld Z, et al. (1999) Thrombophilic polymorphisms are common in women with fetal loss without apparent cause. Thromb Haemost 82: 6-9.

10. Kupferminc MJ, Peri H, Zwang E, Yaron Y, Wolman I, et al. (2000) High prevalence of the prothrombin gene mutation in women with intrauterine growth retardation, abruptio placentae and second trimester loss. Acta Obstet Gynecol Scand 79: 963-967.

11. Gerhardt A, Scharf RE, Beckmann MW, Struve S, Bender HG, et al. (2000) Prothrombin and factor $\mathrm{V}$ mutations in women with a history of thrombosis during pregnancy and the puerperium. N Engl J Med 342: 374-380.

12. Gawish GE (2011) Molecular characterization of factor $V$ leiden $G 1691 A$ and prothrombin G20210A mutations in Saudi newborns with stroke. Biochem Genet 49: 601-610.

13. Ranguelov RD, Rosenthal N, Bromley C, Vasef MA (2002) Detection of factor $\checkmark$ leiden and prothrombin gene mutations in patients who died with thrombotic events. Arch Pathol Lab Med 126: 1193-1196.

14. Rai R, Tuddenham E, Backos M, Jivraj S, El'Gaddal S, et al. (2003) Thromboelastography, whole-blood haemostasis and recurrent miscarriage. Hum Reprod 18: 2540-2543.

15. Carp H, Torchinsky A, Fein A, Toder V (2001) Hormones, cytokines and fetal anomalies in habitual abortion. Gynecol Endocrinol 15: 472-483.

16. Goodman CS, Coulam CB, Jeyendran RS, Acosta VA, Roussev R (2006) Which thrombophilic gene mutations are risk factors for recurrent pregnancy loss? Am J Reprod Immunol 56: 230-236.

17. Coulam CB, Jeyendran RS, Fishel LA, Roussev R (2006) Multiple thrombophilic gene mutations rather than specific gene mutations are risk factors for recurrent miscarriage. Am J Reprod Immunol 55: 360-368.

18. Ridker PM, Miletich JP, Buring JE, Ariyo AA, Price DT, et al. (1998) Factor V Leiden mutation as a risk factor for recurrent pregnancy loss. Ann Intern Med 128: $1000-1003$

19. Raziel A, Kornberg Y, Friedler S, Schachter M, Sela BA, et al. (2001) Hypercoagulable thrombophilic defects and hyperhomocysteinemia in patients with recurrent pregnancy loss. Am J Reprod Immunol 45: 65-71.

20. Zammiti W, Mtiraoui N, Mercier E, Abboud N, Saidi S, et al. (2006) Association of factor $\mathrm{V}$ gene polymorphisms (Leiden; Cambridge; Hong Kong and HR2 haplotype) with recurrent idiopathic pregnancy loss in Tunisia. A case-control study. Thromb Haemost 95: 612-617.

21. Mtiraoui N, Borgi L, Hizem S, Nsiri B, Finan RR, et al. (2005) Prevalence of antiphospholipid antibodies, factor V G1691A (Leiden) and prothrombin G20210A mutations in early and late recurrent pregnancy loss. Eur J Obstet Gynecol Reprod Biol 119: 164-170.

22. Sehirali S, Inal MM, Yildirim Y, Balim Z, Kosova B, et al. (2005) Prothrombin G20210A mutation in cases with recurrent miscarriage: a study of the mediterranean population. Arch Gynecol Obstet 273: 170-173. 
Citation: Gawish G, Al-Khamees O (2013) Molecular Characterization of Factor V Leiden G1691A and Prothrombin G20210A Mutations in Saudi Females with Recurrent Pregnancy Loss. J Blood Disorders Transf 4: 165. doi: 10.4172/2155-9864.1000165

23. Altintas A, Pasa S, Akdeniz N, Cil T, Yurt M, et al. (2007) Factor V Leiden and G20210A prothrombin mutations in patients with recurrent pregnancy loss: data from the southeast of Turkey. Ann Hematol 86: 727-731.

24. Kobashi G, Kato EH, Morikawa M, Shimada S, Ohta K, et al. (2005) MTHFR C677T Polymorphism and factor $V$ Leiden mutation are not associated with recurrent spontaneous abortion of unexplained etiology in Japanese women. Semin Thromb Hemost 31: 266-271.

25. Wolf CE, Haubelt H, Pauer HU, Hinney B, Krome-Caser C, et al. (2003) Recurrent pregnancy loss and its relation to FV Leiden, FII G20210A and polymorphisms of plasminogen activator and plasminogen activator inhibitor. Pathophysiology of Haemostasis Thrombosis. 33:134-137.

26. Rey E, Kahn SR, David M, Shrier I (2003) Thrombophilic disorders and fetal loss: a meta-analysis. Lancet 361: 901-908.

27. Pihusch R, Buchholz T, Lohse $P$, Rübsamen $H$, Rogenhofer $N$, et al. (2001) Thrombophilic gene mutations and recurrent spontaneous abortion: prothrombin mutation increases the risk in the first trimester. Am J Reprod Immunol 46: 124-131.

28. Krause M, Zwinge B, Vigh TH, Scharrer I. (2003) Important role of FV G1691A in women with pregnancy loss without apparent causes. Journal of Thrombosis and Haemostasis 1:12-18.

29. Grandone E, Margaglione M, Colaizzo D, D’Andrea G, Cappucci G, et al. (1998) Genetic susceptibility to pregnancy-related venous thromboembolism: roles of factor $V$ Leiden, prothrombin G20210A, and methylenetetrahydrofolate reductase C677T mutations. Am J Obstet Gynecol 179:1324-1328.
30. Martinelli I, Taioli E, Cetin I, Marinoni A, Gerosa S, et al. (2000) Mutations in coagulation factors in women with unexplained late fetal loss. N Engl J Med 343: 1015-1018.

31. Kovacheva K, Ivanov P, Konova E, Simeonova M, Komsa-Penkova R (2007) [Genetic thrombophilic defects (Factor V Leiden, prothrombin G20210A, MTHFR C677T) in women with recurrent fetal loss]. Akush Ginekol (Sofiia) 46 : 10-16.

32. Foka ZJ, Lambropoulos AF, Saravelos $H$, Karas GB, Karavida A, et al. (2000) Factor $V$ leiden and prothrombin G20210A mutations, but not methylenetetrahydrofolate reductase C677T, are associated with recurrent miscarriages. Hum Reprod 15: 458-462.

33. Sottilotta G, Oriana V, Latella C, Luise F, Piromalli A, et al. (2006) Genetic prothrombotic risk factors in women with unexplained pregnancy loss. Thromb Res 117: 681-684.

34. Glueck CJ, Gogenini S, Munjal J, Tracy T, Pranikoff J, et al. (2008) Factor V Leiden mutation: a treatable etiology for sporadic and recurrent pregnancy loss. Fertil Steril 89: 410-416.

35. Sotiriadis A, Vartholomatos G, Pavlou M, Kolaitis N, Dova L, et al. (2007) Combined thrombophilic mutations in women with unexplained recurrent miscarriage. Am J Reprod Immunol 57: 133-141. 